\title{
NAPHAZOLINE NITRATE, BISACODYL AND GUTTALAX TREAT MICROWAVE SONIC EFFECTS
}

\author{
Robert Skopec \\ Researcher-analyst, Dubnik, Slovakia, \\ (Received on Date: 23rd April 2020 \\ Date of Acceptance: $20^{\text {th }}$ May 2020 \\ Date of Publish: $01^{\text {st }}$ July 2020) \\ Email id: zxcbnvm7@gmail.com
}

\begin{abstract}
At the end of the December 2018 we have found a medicine fully treating the damages caused the Frey Effect of Microvawe and other types of Sonic Weapons in Human's internal, endogenous organs. I am proposing to use Naphasoline nitrate, (former) nasal decongestatnt, to treat Carcinogenesis of the Human's internal, endogenous organs caused by Sonic Weapons through the release and cleaning of the Lymphatic ways in patients with colorectal, colon, pancreatic, breast, etc., cancer. I have proved this healing effect of the Naphazoline nitrate on Myself during treatment in last months of the year 2018. Naphazoline Nitrate, Bisacodyl and Guttalax are clearing the Pylorus in human digestive system and treat so the Microwave Sonic Effects.
\end{abstract}

\section{Keywords}

Nasal decongestatnt, naphazoline nitrate, the Frey effect, the acoustic effect of microwaves, the sonic delusions, bio-acoustic deterrents, sonic weapons, carcinogenesis, loss of hearing, balance, brain injuries, nausea, headaches, ear-ringing, carcinogenesis of human's internal organs, pancreatic cancer, colon cancer, colorectal cancer.

\section{Conflict of Interests}

The author declares no conflict of interests. 


\section{INTRODUCTION}

Exposure to microwave, infrasound, etc., sonic weapons has been demonstrated to affects recipients with symptoms including fear, sorrow, depression, anxiety, nausea, chest pressure and hallucination. It can cause objects to move through vibration and the body's internal organs can be affected. $(1,2)$

\section{Materials and Methods}

The term infrasonic applied to sound refers to sound waves below the frequencies of audible sound. Nominally includes frequencies under $20 \mathrm{~Hz}$. Sources of infrasound in nature includes volcanoes, avalanches, earthquakes and meteorites.

The healthy human ear can hear frequencies ranging from $20 \mathrm{~Hz}$ to $20,000 \mathrm{~Hz}$. Over time, the hair-like stereocilia may get damaged or broken. If enough of them are damaged, hearing loss results. The high frequency area of the cochlea is often damaged by loud sound.

Infrasound is sound which extends below the range of human hearing (from $20 \mathrm{~Hz}$ to $0.001 \mathrm{~Hz}$ ), and it emitates from many natural and man-made sources. For example, some animals, such as whales, elephants and giraffes communicate using infrasound over long distances.

Sonic and Ultrasonic weapons (USW) are weapons of various types that use sound to injure, incapacitate, or kill a target. New personal communications shows that infrasound can cause trough vibrations, resonance frequency about 7 $\mathrm{Hz}$ with internal organs of humans cause also cancer, such as colorectal cancer, colon cancer, pancreatic cancer, etc.

\section{$(2,3,4)$}

Some of these weapons have been described as sonic bullets, sonic grenades, sonic mines, or sonic cannons. Some USWs make a focused beam of sound or ultrasound, some made an area field of sound.

As an example of used sonic weapon we can use The LRAD Sound Cannon as an acoustic weapon and communication device. Developed by the LRAD Corporation to broadcast messages and pain-inducing deterrent tones over long distances. LRAD devices come in various iterations that produce varying degrees of sound. They can be mounted to a vehicle or handheld.

Protests in Fergusson, Missouri have reached a terrifying fever pitch, and the ludicrously armed Fersguson Police Department is bringing all its crowdcontrol weapons to bear, tear gas, stun grenades, rubber bullets. One of the most controversial of those is the LRAD Sound Cannon. The device produces a sound that can be directed in a beam up to 30degress wide, and the military-grade LRAD 2000X can transmit voice commands at up to $162 \mathrm{~dB}$ up to 5.5 miles away.

The LRAD Corporation says that anyone within a 100 meters of the device's sound path will experience an extreme pain. The version generally utilized by Police Departments (the LRAD 500X) is designed to communicate at up to 2000 meters during ideal conditions. In a typical outdoor environment, the device can be heard for 650 meters. The 500X version is also capable of short bursts of directed sound that cause headaches in anyone 
within a 300 meter range. Anyone within 15 meters of the device's audio path can experience permanent hearing loss. LRAD claims the device is not a weapon, but a directed-sound communication device. $(3,4)$

\section{Developing a new class of weapons}

Allan H. Frey, has lived at his home outside Washington. In 1960, he stumbled on an acoustic effect of microwaves that was eventually named after him. Microwaves are ubiquitous in modern life. The short radio waves power radars, cook foods, relay messages and link cellphones to antenna towers. They're a form of electromagnetic radiation on the same spectrum as light and X-rays, only at the opposite end.

While radio broadcasting can employ waves a mile or more in length, microwaves range in size from roughly a foot to a tiny fraction of an inch. They're seen as harmless in such everyday uses as microwaving foods. But their diminutive size also enables tight focusing, as when dish antennas turn disorganized rays into concentrated beams.

The dimensions of the human head, scientists say, make it a fairly good antenna for picking up microwave signals. Mr. Frey, a biologist, said he stumbled on the acoustic effect in 1960 while working for General Electric's Advanced Electronics Center at Cornell University. A man who measured radar signals at a nearby G.E. facility came up to him at a meeting and confided that he could hear the beam's pulses - zip, zip, zip. Intrigued, Mr. Frey traveled to the man's workplace in
Syracuse and positioned himself in a radar beam. "Lo," he recalled, "I could hear it, too."

Mr. Frey's resulting papers - reporting that even deaf people could hear the false sounds - founded a new field of study on radiation's neural impacts. Mr. Frey's first paper, in 1961, reported that power densities 160 times lower than "the standard maximum safe level for continuous exposure" could induce the sonic delusions. His second paper, in 1962 , pinpointed the brain's receptor site as the temporal lobes, which extend beneath the temples. Each lobe bears a small region - the auditory cortex - that processes nerve signals from the outer and inner ears. Investigators raced to confirm and extend Mr. Frey's findings. At first they named the phenomenon after him, but eventually called it the microwave auditory effect and, in time, more generally, radiofrequency hearing.

\section{Tinnitus: Ringing in the ears and what to do about it}

Tinnitus (pronounced tih-NITE-Us or TIN-ihtus) is sound in the head with no external source. For many, it's a ringing sound, while for others, it's whistling, buzzing, chirping, hissing, humming, roaring, or even shrieking. The sound may seem to come from one ear or both, from inside the head, or from a distance. It may be constant or intermittent, steady or pulsating. $(3,4)$

Almost everyone has had tinnitus for a short time after being exposed to extremely loud noise. For example, 
attending a loud concert can trigger short-lived tinnitus. Some medications (especially aspirin and other nonsteroidal anti-inflammatory drugs taken in high doses) can cause tinnitus that goes away when the drug is discontinued. When it lasts more than six months, it's known as chronic tinnitus. As many as 50 to 60 million people in the United States suffer from this condition; it's especially common in people over age 55 and strongly associated with hearing loss. Many people worry that tinnitus is a sign that they are going deaf or have another serious medical problem, but it rarely is.

Most tinnitus is subjective, meaning that only you can hear the noise. But sometimes it's objective, meaning that someone else can hear it, too. For example, if you have a heart murmur, you may hear a whooshing sound with every heartbeat; your clinician can also hear that sound through a stethoscope. Some people hear their heartbeat inside the ear - a phenomenon called pulsatile tinnitus. It's more likely to happen in older people, because blood flow tends to be more turbulent in arteries whose walls have stiffened with age. Pulsatile tinnitus may be more noticeable at night, when you're lying in bed and there are fewer external sounds to mask the tinnitus. If you notice any new pulsatile tinnitus, you should consult a clinician, because in rare cases it is a sign of a tumor or blood vessel damage.

The course of chronic tinnitus is unpredictable. Sometimes the symptoms remain the same, and sometimes they get worse. In about $10 \%$ of cases, the condition interferes with everyday life so much that professional help is needed.

While there's no cure for chronic tinnitus, it often becomes less noticeable and more manageable over time. You can help ease the symptoms by educating yourself about the condition - for example, understanding that it's not dangerous. There are also several ways to help tune out the noise and minimize its impact.

\section{Auditory pathways and tinnitus}




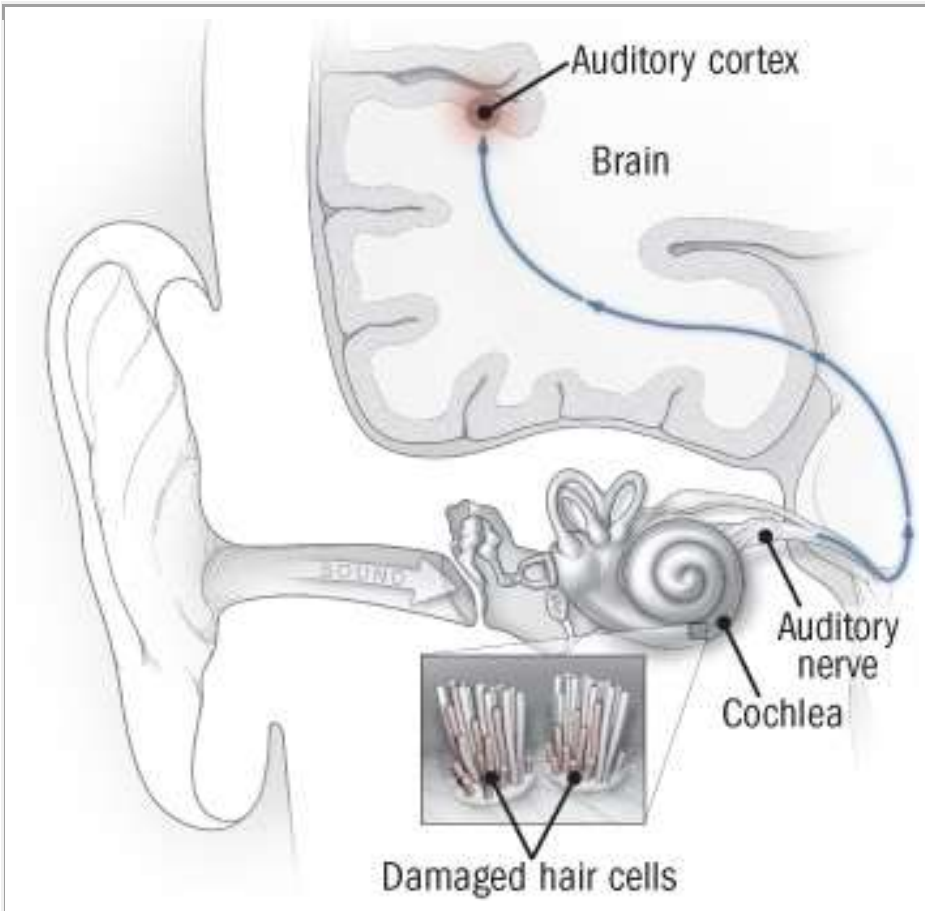

Sound waves travel through the ear canal to the middle and inner ear, where hair cells in part of the cochlea help transform sound waves into electrical signals that then travel to the brain's auditory cortex via the auditory nerve. When hair cells are damaged - by loud noise or ototoxic drugs, for example - the circuits in the brain don't receive the signals they're expecting. This stimulates abnormal activity in the neurons, which results in the illusion of sound, or tinnitus.

\section{What's going on?}

Most people who seek medical help for tinnitus experience it as subjective, constant sound like constant ringing in the ears or a buzzing sound in the ear, and most have some degree of hearing loss. Things that cause hearing loss (and tinnitus) include loud noise, medications that damage the nerves in the ear (ototoxic drugs), impacted earwax, middle ear problems (such as infections and vascular tumors), and aging. Tinnitus can also be a symptom of Meniere's disease, a disorder of the balance mechanism in the inner ear.

Tinnitus can arise anywhere along the auditory pathway, from the outer ear through the middle and inner ear to the brain's auditory cortex, where it's thought to be encoded (in a sense, imprinted). One of the most common causes of tinnitus is damage to the hair cells in the cochlea (see "Auditory pathways and tinnitus"). These cells help transform sound waves into nerve signals. If the auditory pathways or circuits in the brain don't receive the signals they're expecting from the cochlea, the brain in effect "turns up the gain" on those pathways in an effort to detect the signal — in much the same way that you turn up the volume on a car radio when you're trying to find a station's signal. The resulting electrical noise takes the form of tinnitus - a sound that is highpitched if hearing loss is in the highfrequency range and low-pitched if it's in the low-frequency range. This kind of 
tinnitus resembles phantom limb pain in an amputee - the brain is producing abnormal nerve signals to compensate for missing input.

Most tinnitus is "sensorineural," meaning that it's due to hearing loss at the cochlea or cochlear nerve level. But tinnitus may originate in other places. Our bodies normally produce sounds (called somatic sounds) that we usually don't notice because we are listening to external sounds. Anything that blocks normal hearing can bring somatic sounds to our attention. For example, you may get head noise when earwax blocks the outer ear.

\section{Some drugs that can cause or worsen tinnitus}

$\underline{\text { Aspirin }}$ and other nonsteroidal anti-inflammatory drugs, including ibuprofen (Motrin) and naproxen (Aleve, Naprosyn)

Certain antibiotics, including ciprofloxacin (Cipro), doxycycline (Vibramycin, others), gentamicin (Garamycin), erythromycin (Ery-Tab, others), tetracycline (Sumycin), tobramycin (Nebcin), and vancomycin (Vancocin)

Antimalarial drugs such as chloroquine and quinine

Certain anticonvulsants, including carbamazepine (Tegretol, others) and valproic acid (Depakote, others)

Certain cancer drugs, including cisplatin (Platinol) and vincristine (Oncovin, Vincasar)

Loop diuretics (when given intravenously in high doses), including bumetanide (Bumex), furosemide (Lasix), and torsemide (Demadex)

Tricyclic antidepressants such as amitriptyline (Elavil, others), clomipramine (Anafranil), and imipramine (Tofranil)

\section{Evaluate and treat underlying problems}

If you develop tinnitus, it's important to see your clinician. She or he will take a medical history, give you a physical examination, and do a series of tests to try to find the source of the problem. She or he will also ask you to describe the noise you're hearing (including its pitch and sound quality, and whether it's constant or periodic, steady or pulsatile) and the times and places in which you hear it. Your clinician will review your medical history, your current and past exposure to noise, and any medications or supplements you're taking. Tinnitus can be a side effect of many medications, especially when taken at higher doses (see "Some drugs that can cause or worsen tinnitus").
Musculoskeletal factors - jaw clenching, tooth grinding, prior injury, or muscle tension in the neck - sometimes make tinnitus more noticeable, so your clinician may ask you to tighten muscles or move the jaw or neck in certain ways to see if the sound changes. If tight muscles are part of the problem, massage therapy may help relieve it. $(5,6)$

Tinnitus that's continuous, steady, and high-pitched (the most common type) generally indicates a problem in the auditory system and requires hearing tests conducted by an audiologist. Pulsatile tinnitus calls for a medical evaluation, especially if the noise is frequent or constant. MRI or CT imaging may be needed to check for a tumor or blood vessel abnormality. 
Your general health can affect the severity and impact of tinnitus, so this is also a good time to take stock of your diet, physical activity, sleep, and stress level - and take steps to improve them. You may also be able to reduce the impact of tinnitus by treating depression, anxiety, insomnia, and pain with medications or psychotherapy.

If you're often exposed to loud noises at work or at home, it's important to reduce the risk of hearing loss (or further hearing loss) by using protectors such as earplugs or earmuff-like or custom-fitted devices.

\section{Selected resources}

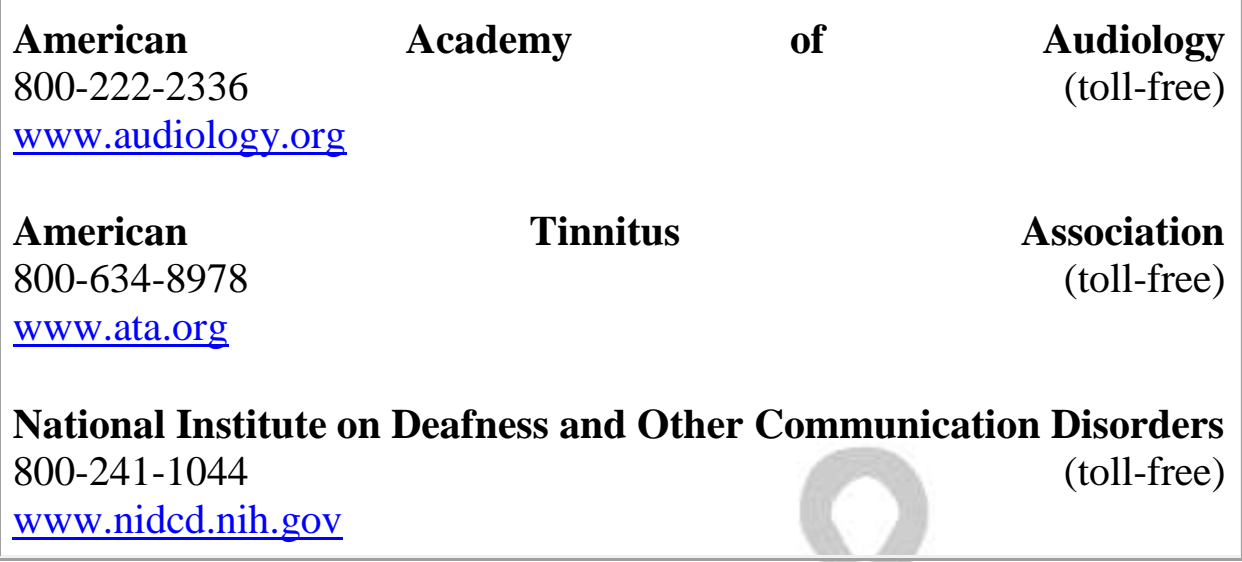

\section{Managing tinnitus}

In addition to treating associated problems (such as depression or insomnia), there are several strategies that can help make tinnitus less bothersome. No single approach works for everyone, and you may need to try various combinations of techniques before you find what works for you. If you have age-related hearing loss, a hearing aid can often make tinnitus less noticeable by amplifying outside sounds.

There is no FDA-approved drug treatment for tinnitus, and controlled trials have not found any drug, supplement, or herb to be any more effective than a placebo. That includes ginkgo biloba, which is sometimes promoted for this purpose. Some patients believe that acupuncture helps, but it too has been found to be no better than a placebo. $(7,8)$
The most effective approaches are behavioral strategies and soundgenerating devices, often used in combination. They include the following:

Cognitive behavioral therapy (CBT). CBT uses techniques such as cognitive restructuring and relaxation to change the way patients think about and respond to tinnitus. Patients usually keep a diary and perform "homework" to help build their coping skills. Therapy is generally short-term - for example, weekly sessions for two to six months. A 2010 review of six studies by the Cochrane Collaboration (an international group of health authorities who evaluate randomized trials) found that after CBT, the sound was no less loud, but it was significantly less bothersome, and patients' quality of life improved.

Tinnitus retraining therapy (TRT). This technique is based on the assumption that tinnitus results from abnormal 
neuronal activity (see "What's going on?"). The aim is to habituate the auditory system to the tinnitus signals, making them less noticeable or less bothersome. The main components of TRT are individual counseling (to explain the auditory system, how tinnitus develops, and how TRT can help) and sound therapy. A device is inserted in the ear to generate low-level noise and environmental sounds that match the pitch, volume, and quality of the patient's tinnitus. Depending on the severity of the symptoms, treatment may last one to two years.

When TRT was developed in the 1980s by neuroscientist Dr. Pawel Jastreboff (now at Emory University in Atlanta), it was designed to be administered according to a strict protocol. Today, the term TRT is being used to describe modified versions of this therapy, and the variations make accurate assessment of its effectiveness difficult. Individual studies have reported improvements in as many as $80 \%$ of patients with high-pitched tinnitus. In a Cochrane review of the one randomized trial that followed Jastreboff's protocol and met the organization's standards, TRT was much more effective in reducing tinnitus severity and disability than a technique called masking (see below). $(9,10)$

Masking. Masking devices, worn like hearing aids, generate low-level white noise (a high-pitched hiss, for example) that can reduce the perception of tinnitus and sometimes also produce residual inhibition - less noticeable tinnitus for a short time after the masker is turned off. A specialized device isn't always necessary for masking; often, playing music or having a radio, fan, or white-noise machine on in the background is enough. Although there's not enough evidence from randomized trials to draw any conclusions about the effectiveness of masking, hearing experts often recommend a trial of simple masking strategies (such as setting a radio at low volume between stations) before they turn to more expensive options.

\section{Biofeedback and stress management.}

Tinnitus is stressful, and stress can worsen tinnitus. Biofeedback is a relaxation technique that helps control stress by changing bodily responses. Electrodes attached to the skin feed information about physiological processes such as pulse, skin temperature, and muscle tension into a computer, which displays the output on a monitor. Patients learn how to alter these processes and reduce the body's stress response by changing their thoughts and feelings. Mindfulnessbased stress reduction techniques may also help.

Other therapies. Other treatments that have been studied for tinnitus include transcutaneous electrical stimulation of parts of the inner ear by way of electrodes placed on the skin or acupuncture needles, and stimulation of the brain using a powerful magnetic field (a technique called repetitive transcranial magnetic stimulation, or rTMS). Transcutaneous electrical stimulation has been shown to be no more effective than a placebo. In two small trials, rTMS compared with a sham procedure helped improve the perception of tinnitus in a few patients. 


\section{A solution for constant ringing in your ear}

It might not work for everyone, but it could mean precious silence for a lot of tinnitus patients. $(11,12)$

Apparently, around 2 million Americans can't work because of tinnitus, and it's also the most common serviceconnected disability in veterans. The condition doesn't have a cure yet, but those suffering from it might not have to endure all the phantom ringing, clicking and hissing for life, thanks to a device developed by researchers from the University of Michigan. Their creation treats tinnitus by using precisely timed sounds and weak electrical pulses designed to persuade damaged nerves in the region of the brainstem called dorsal cochlear nucleus into working correctly again.

Team leader and U-M Medical School professor Susan Shore explained:

"When the main neurons in [the dorsal cochlear nucleus], called fusiform cells, become hyperactive and synchronize with one another, the phantom signal is transmitted into other centers where perception occurs. If we can stop these signals, we can stop tinnitus. That is what our approach attempts to do..."

The team tested the small, box-like device on guinea pigs first before testing it on 20 tinnitus patients who used it 30 minutes every day for four weeks. It played a sound into the ears and alternated it with precisely timed, mild electrical pulses to the cheek or neck for half the patients. The other half only received sounds. They found that the loudness of the phantom sounds decreased by 12 decibels in some of the patients who received both sounds and electrical pulses. A couple of subjects even said their tinnitus disappeared completely. However, those who only received sounds didn't report a change in their condition.

Due to the way their device works, it can only treat somatic tinnitus. People who have this variant can modulate the phantom ringing/hissing they hear by pressing a part of their face and forehead or clenching their jaw. Thankfully, studies say two-thirds of tinnitus patients suffer from somatic forms of the condition, making their device a promising treatment for most people. The researchers are trying to find a way to make it work just as well for nonsomatic patients, though. They're also conducting more studies and tests to figure out how to make its effects last longer, since the testers' symptoms came back after a couple of weeks.

Shore said:

"We're definitely encouraged by these results, but we need to optimize the length of treatments, identify which subgroups of patients may benefit most, and determine if this approach works in patients who have nonsomatic forms of the condition that can't be modulated by head and neck maneuvers."

\section{Results}

Naphasoline Nitrate, Guttalax and Dulcolax treat microwave sonic weapons

The use of naphazoline nitrate may result in an analgesic effect upon first use, 
through activation of adrenergic and opioidergic systems, followed by a promigraine effect via a late induction of an inflammatory cascade, modulated by nitric oxide and arachidonic acid. The observation that naphazoline detoxification relieved the patient's headache, indicates that prolonged use of naphazoline may cause chronic headaches. Therefore, physicians should ask for details on the use of nasal decongestants in patients complaining of chronic headache, as they could potentially be suffering from a headache caused be the Frey effect of Sonic Weapons use against him. $(3,4)$

\section{The headache experienced by the patient was bilateral, throbbing,} sometimes very severe, and associated with nausea, vomiting, photophobia, osmophobia and worsened by head movements.

The patient had been treated by his general practicioner (GP) with medication to relieve the headache (metamizole, rizatriptan, zolmitriptan, acetylsalicylic acid, nimesulide, ibuprofen, naproxen sodium), consumed more than once a day, until the age of 24. Since then, he had stopped the consumption of such painkillers because he found that naphazoline nitrate nasal spray was more effective. He began with 2 shots $(0,14 \mathrm{mg}$ of drug for any shot) for each nostril 3 times a day, and at the time of presentation, due to pain recurrence, was using the spray 5-6 times a day, and experiencing immediate, yet temporary, relief of the symptoms every time. When asked, the patient said that he had used naphazoline nitrate nasal spray on an occasional basis since the age of 16, in order to self-medicate for self-diagnosed chronic rhinitis.

The chronic headache of the Frey effect disappeared following the treatment suggested by otolaryngologist. After three months of headache diary recording and clinical re-evaluation, the clinical picture was dramatically changed: patient presented only sporadic attacks of migraine without aura (ICHD-II 1.1). By an anamnesis re-evaluation it emerged that an episodic headache arose in childhood and worsen over the years, until it became chronic by the age of 18 . One year after naphazoline detoxification, the patient has suffered from a few attacks during the year, treated with triptans.

Naphazoline is a sympathomimetic drug, an imidazolinic derivate with marked alpha-adrenergic activity. It enhances the release of noradrenaline from adrenergic termination, immediately relieving the nasal congestion thanks to its vasoconstricting action on the vessels of nasal mucosa. Because of its adrenergic activity, this drug can also produce adverse effects, like rhinitis medicamentosa, hypertension, headache and acute depression of central nervous system with marked sedation. Moreover, cases of ischemic and hemorrhagic stroke secondary to naphazoline have also been reported, mediated by the alphal and alpha2 adrenergic vasoconstrictive effect that is also exerted on brain vessels.

Naphazoline can trigger headaches because of its adrenergic activity Alphal receptors are associated with G-proteins that generate a cascade of events 
leading to the production of arachidonic acid (AA) and nitric oxide (NO). AA and $\mathrm{NO}$ release leads to a late inflammatory vasodilatation that could induce a migraine attack. Moreover, it is also possible that the same naphazoline nitrate, consumed by our patient, could have contributed to the production and release of further NO by a chemical reduction of the naphazoline salt. In fact, $\mathrm{NO}$ donors are currently used to induce migraine attacks in clinical and experimental settings.

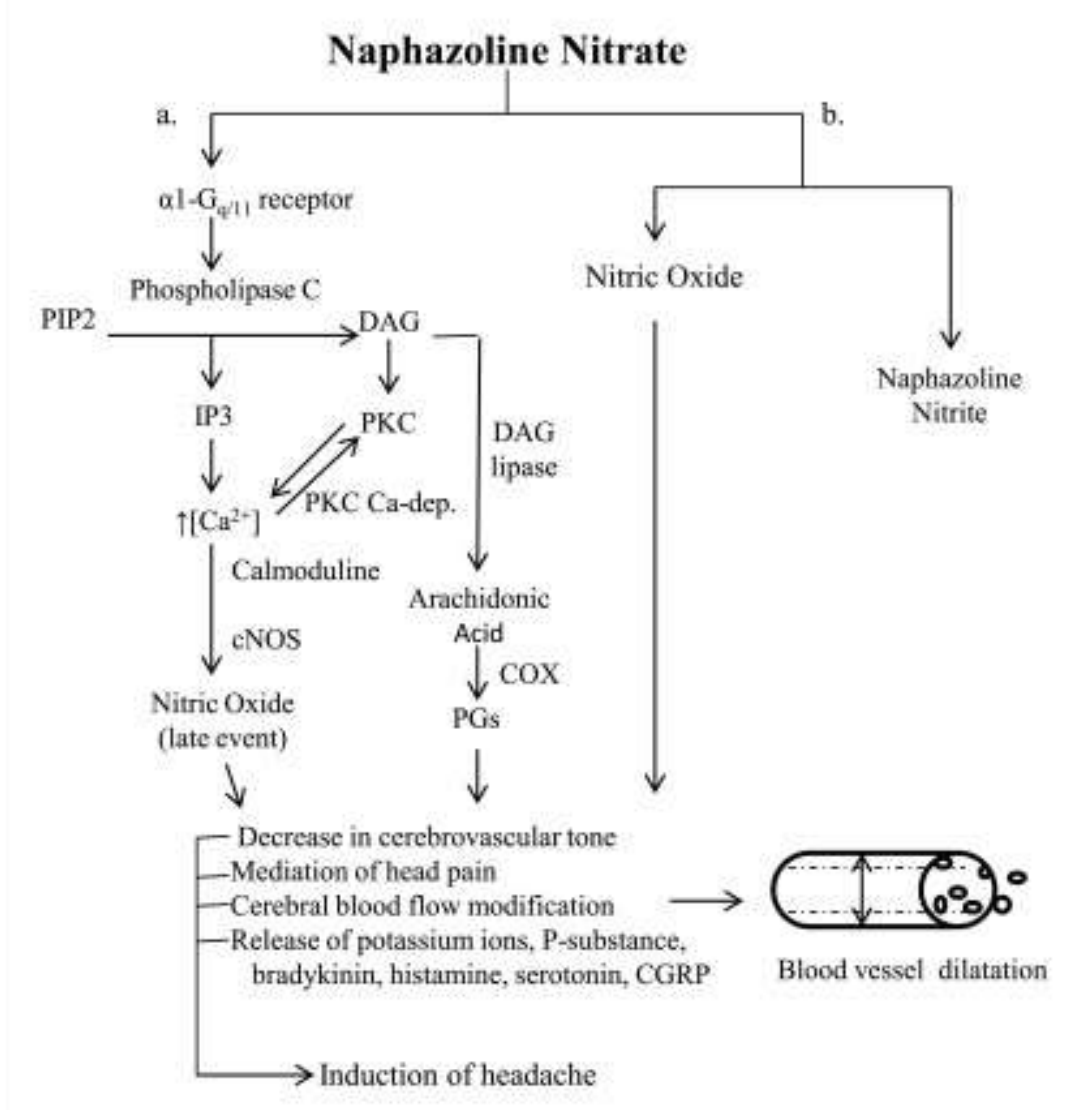

Figure 1.

\section{Events induced by naphazoline caused by the Frey effect's headache.}

a. Naphazoline activates al receptors that are associated with G-proteins that cause the activation of phospholipase $C$. Phospholipase C cleavesphosphatidylinositol 4,5-bisphosphate (PIP2) into two second messengers, inositol 1,4,5trisphosphate (IP3) and diacylglycerol (DAG) which in turn cause an increase in the level of calcium and protein kinase $C$. Diacylglycerol, by DAG lipase, takes part in the creation of arachidonic acid which is a precursor in the production of prostaglandins (PGs), mediated by cyclooxygenase (COX). The higher concentration of intracellular calcium allows an increase in the complex of calcium-calmodulin and therefore the activation of constitutive nitrous oxide synthetase (CNOS) with the generation of nitric oxide. b. Naphazoline nitrate can contribute to the production and release of further NO by a chemical reduction of the naphazoline salt. Prostaglandins and nitric oxide contribute to the activation of nociceptors and the transmission of the 
pain pulse from the periphery to the centre. Consequently there is a release of substances such as potassium ions, Psubstance, bradykinin, histamine, serotonin and CGRP that keep nociceptors active and result in vasodilatation and extravasation of plasma proteins from the vessels. (7-12)

However, despite the late effect as a migraine trigger factor, naphazoline might also have an early action as an anti-migraine agent acting on alpha receptors of muscle, immune cells, Locus
Coeruleus and spinal cord. In fact, alpha2 adrenergic receptors have a peripheral analgesic effect, thanks to the activation of opioidergic receptors, via endogenous opioid release. Moreover, both alphal and alpha2 adrenergic receptors agonists have been proposed to be specifically useful for the treatment of migraine by mechanisms that mediate the early vasoconstrictive effect related to their activation, similarly to triptans that carry out an analgesic action through the serotoninergic agonism that also mediates vasoconstriction.

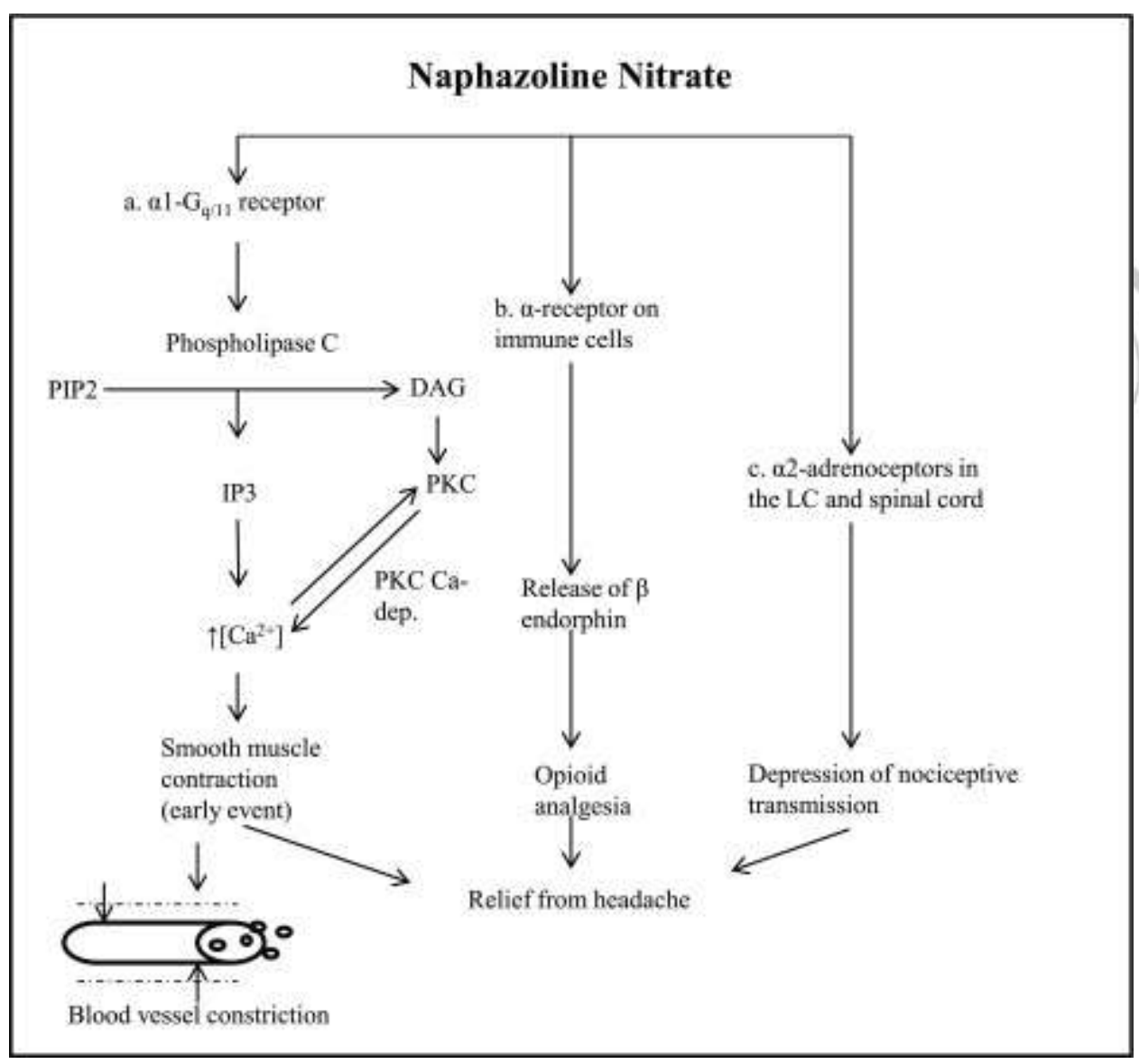

Figure 2.

\section{Possible early events that induce peripheral analgesic effect.}

a. Naphazoline activates alphal a smooth muscle contraction due to an receptors that are associated with Gproteins that cause the activation of phospholipase C. Phospholipase C cleaves phosphatidyl-inositol 4,5bisphosphate (PIP2) into inositol 1,4,5increase of intracellular calcium. b. Alpha-Receptors on immune cells release $\beta$-endorphins that cause opioid analgesia. c. Activation of alpha2 trisphosphate (IP3). Consequently there is adrenoceptors in the Locus Coeruleus 
and spinal cord provokes the depression of nociceptive transmission.

We could therefore presume that in our patient, in a similar manner to the "triptans effect", naphazoline could exert an antimigraine action but also induce a rebound chronic headache due to medication overuse and/or a proinflammatory cytokine-mediated headache induction. Indeed, our patient had experienced such a sudden analgesic effect; otherwise he would not have continued to take naphazoline. (13, 14)

Bisacodyl (INN) is an organic compound that is used as a stimulant laxative drug. It works directly on the colon to produce a bowel movement. It is typically prescribed for relief of episodic and chronic constipation and for the management of neurogenic bowel dysfunction, as well as part of bowel preparation before medical examinations, such as for a colonoscopy.

Bisacodyl is a derivative of triphenylmethane. It was first used as a laxative in 1953 because of its structural similarity to phenolphthalein.

Bisacodyl is marketed under the trade names Dulcolax/Durolax, Muxol, Fleet, Nourilax, Alophen, Correctol, and Carter's Little Pills (formerly Carter's Little Liver Pills), as well as being available generically. It is usually sold as $5 \mathrm{mg}$ tablets, $10 \mathrm{mg}$ suppositories, or $5 \mathrm{mg}$ pediatric suppositories. It is also available as a 1.25 US fluid ounces $(37 \mathrm{ml})$ pre-packaged enema containing a $10 \mathrm{mg}$ delivered dose of liquid bisacodyl.
When bisacodyl is administered orally, it is usually taken at bedtime. Oral administration is known to produce no action for more than eight hours and then to work suddenly and relatively quickly. This is especially true if more than $10 \mathrm{mg}$ is taken at one time. Normally, the dosage is 5 or $10 \mathrm{mg}$, but up to $30 \mathrm{mg}$ can be taken for complete cleansing of the bowel before a procedure. $(15,16)$

When administered rectally in suppository form, it is usually effective in 15 to 60 minutes. For optimal use, if used as a suppository, it is recommended that bisacodyl be given after breakfast to synchronize with the gastrocolic reflex. Two suppositories can be inserted at once if a very strong, purgative, enemalike result is needed. A few hours after the initial evacuation, there can be a secondary action which will continue as long as there is unexpelled bisacodyl present in the rectum. As a commercially prepared micro-enema, it is usually effective in 5 to 20 minutes.

Bisacodyl works by stimulating enteric nerves to cause peristalsis, i.e., colonic contractions. It is also a contact laxative; it increases fluid and salt secretion. The action of bisacodyl on the small intestine is negligible; stimulant laxatives mainly promote evacuation of the colon.

\section{Guttalax Dosage form, composition and packaging}

Drops for internal use in the form of a clear, slightly viscous solution, from colorless to slightly yellowish or slightly yellowish brown. 


\section{Guttalax Pharmacological action}

Laxative. The active ingredient - sodium picosulphate - is a laxative of the triarylmethane group.As a locally acting laxative, sodium picosulphate after bacterial cleavage in the colon has a stimulating effect on the mucosa of the colon, increasing motility, and promotes accumulation of water and electrolytes in the colon. This leads to the stimulation of defecation, reduction of evacuation time and stool softening. In case of simultaneous use of Guttalax in high doses and diuretics or GC the risk of electrolyte imbalance (hypokalemia) is increased. Simultaneous administration of antibiotics may reduce the laxative effect of Guttalax. Electrolyte imbalance may increase sensitivity to cardiac glycosides. Symptoms: in case of high doses administration there may be diarrhea, dehydration, decreased blood pressure, disruption of water and electrolyte balance, hypokalemia, cramps. In addition, there were reports of ischemia of the colon associated with administering Guttalax in doses greatly exceeding recommended ones for routine treatment of constipation. $(17,18)$

Guttalax, like other laxatives, in case of a chronic overdose may cause chronic diarrhea, abdominal pain, hypokalemia, secondary hyperaldosteronism, urolithiasis. Due to the chronic laxative abuse renal tubular damage, metabolic alkalosis and muscle weakness associated with hypokalemia may develop.
As a laxative in the following cases:

- constipation due to atony and hypotonia of the colon (including in the elderly, in bedridden patients, after surgery, after childbirth and during lactation);

- constipation caused by the administration of drugs;

- to regulate the stool in case of hemorrhoids, proctitis, anal fissures (for softer stool consistency);

- gallbladder diseases, constipationpredominant irritable bowel syndrome;

- constipation caused by intestinal dysbacteriosis, diet issues.

During the II and III trimester of pregnancy (the same is true about other laxatives) the drug can only be prescribed by a doctor. Active metabolite and its glucuronides are not excreted in breast milk. Thus, the drug can be used during breastfeeding.

Treatment: to reduce the absorption of the drug after ingestion one can induce vomiting or perform gastric lavage. Fluid replacement and correction of electrolyte balance may be required, as well as prescription of antispasmodics. It is not recommended to take the drug every day without consulting a doctor for more than 10 days. Long term administration of the drug in high doses can lead to dehydration, electrolyte imbalance, hypokalemia.

In case of simultaneous use of Guttalax in high doses and diuretics or GC the risk of 
electrolyte imbalance (hypokalemia) is increased. Simultaneous administration of antibiotics may reduce the laxative effect of Guttalax. Electrolyte imbalance may increase sensitivity to cardiac glycosides. Data on reliable and well-controlled studies in pregnant women are not available. Long experience in the application did not reveal any adverse effects of the drug on pregnancy. Taking the drug in the I trimester of pregnancy is contraindicated. Studies on the effect of the drug on fertility have not been conducted. Preclinical studies did not reveal any teratogenic effects on reproduction.

\section{Conclusion}

I am proposing to use Naphazoline Nitrate, Guttalax and Dulcolax to treat Carcinogenesis of the Human's internal, endogenous organs caused by Sonic Weapons through the release and cleaning of the Lymphatic ways in patients with colorectal, colon, pancreatic, breast, etc., cancer. I have proved this healing effect of the Naphazoline nitrate on Myself during treatment in last months of the year 2018. Naphazoline Nitrate, Bisacodyl and Guttalax are clearing the Pylorus in human digestive system and treat so the Microwave Sonic Effects.

\section{ACKNOWLEDGMENTS}

The author gratefully acknowledge the assistance of Dr. Marta Ballova, Ing. Konrad Balla, Livuska Ballova and Ing. Jozef Balla.

\section{References}

(1)Myers, More Americans Evacuated From Chins Over Mysterious Ailments, N.Y. Times (June 30, 2018). See also previous posts to dwkcommentaries.com: U.S. Establishes Task Force To Coordinate Response to Health Problems of U.S. Diplomats in Cuba and China (June 5, 2018); Comment: More U.S. Diplomats Report Illness in China (June 6, 2018);Comment: China Pledges to Investigate Sonic Attacks on U.S. Diplomats (June 7, 2018); Comment: U.S. Broadens Health Alert to All Americans in China (June 8, 2018).

(2) See posts to dwkcommentaries.com listed in the "U.S. Diplomats Medical Problems in Cuba, 2017-18" section of List of Posts to dwkcommentaries-Topical: CUBA.

U.S. Embassy \& Consulates in China, Secretary Pompeo's Call with Chinese State Councilor and Foreign Minister Wang Yi (June 28, 2018).

(3) Skopec R: "Darwinian Selection Transforms Organisms to Bio-Robots", World Journal of Biology and Medical Sciences, 2018. -

Z - Skopec - Darwinian selection - 57-77$\underline{16.5 .2018}$

(4) Skopec R: "Science Has a Solution for Sonic Weapons Caused Cancer", Research Trends in Hematology and Oncology, 2018. - $\underline{\text { z - Skopec - RTHO - }}$ $\underline{101-6.6 .2018}$

(5) ERA GLONASS is the modern Russian system of emergency response, similar to the European standard eCall/El 12. The system is designed for use with the Russian 
global satellite navigation system GLONASS.

(6)http://www.foxnews.com/politics/2018 106/07/us-brings-home-group-diplomats-

from-chinaafter-mysterious-illnessreport

(7) Nappi G, Perrotta A, Rossi P, et al. : Chronic daily headache. Expert Rev Neurother. 2008;8(3):361-84 10.1586/14737175.8.3.361

[PubMed] [CrossRef]

(8) International Classification of Disorders, Committee of the International Headache Society. Classification and diagnosis criteria for headache disorders, cranial neuralgia and facial pain. Cephalalgia. 2004;24(Suppl 1):1-150 Reference Source

(9) Johnson DA, Hricik JG: The pharmacology of alpha-adrenergic decongestants.

Pharmacotherapy. 1993;13(6 Pt 2):110S-115S- 143S-146S. 10.1002/j.1875-9114.1993.tb02779.x [PubMed] [CrossRef]

(10) Willems EW, Valdivia LF, Villalón CM, et al. : Possible role of alphaadrenoceptor subtypes in acute migraine therapy. Chephalalgia. 2003;23(4):245$257 \quad 10.1046 / j .1468-2982.2003 .00547 . x$ [PubMed] [CrossRef]

(11) Munaron L: Shuffling the cards in signal transduction: Calcium, arachidonic acid and mechanosensitivity. World J Biol Chem. $2011 ; 2(4): 59-66$ 10.4331/wjbc.v2.i4.59 [PMC free article] [PubMed] [CrossRef]
(12) Romero TR, de Castro Perez A, de Francischi JN, et al. : Probable involvement of alpha(2C)-adrenoceptor subtype and endogenous opioid peptides in the peripheral antinociceptive effect induced by xylazine. Eur J Pharmacol. 2009;608(13):23-7 10.1016/j.ejphar.2009.02.019 [PubMed] [CrossRef]

(13) Wexner, S. D.; Beck, D. E.; Baron, T. H.; Fanelli, R. D.; Hyman, N.; Shen, B.; Wasco, K. E.; American Society of Colon and Rectal Surgeons; American Society for Gastrointestinal Endoscopy; Society of American Gastrointestinal and Endoscopic Surgeons (2006). "A consensus document on bowel preparation before colonoscopy: prepared by a task force from the American Society of Colon and Rectal Surgeons (ASCRS), the American Society for Gastrointestinal Endoscopy (ASGE), and the Society of American Gastrointestinal and Endoscopic Surgeons (SAGES)". Gastrointestinal Endoscopy. $\quad 63 \quad$ (7): 894-909. doi:10.1016/j.gie.2006.03.918. ISSN $0016-$ 5107. PMID 16733101.

(14) Wald, A (January 2016). "Constipation: Advances in Diagnosis and Treatment". JAMA (Review). 315 (2): 18591. $\quad$ doi:10.1001/jama.2015.16994. $\underline{\text { PMID }} 26757467$.

(15) Stiens SA; Luttrel W; Binard JE (November 1998). "Polyethylene glycol versus vegetable oil based bisacodyl suppositories to initiate side-lying bowel care: a clinical trial in persons with spinal cord injury". Spinal Cord. 36 (11): 777-81. doi:10.1038/sj.sc.3100702. PMID 9848486. 
(16) Evans, I. Lynn, "Methods and techniques: The use of Bisacodyl suppositories in preparation for sigmoidoscopy", Gut, British Medical Journal, 1964, 5, 271

(17) "Medicine: Cut Out the Liver". Time. 1951-04-16. Retrieved 2010-04-26.
(18) Robert Engelhorn, Ernst Seeger and Jan H. Zwaving "Laxatives" in Ullmann's Encyclopedia of Industrial Chemistry, Wiley-VCH, Weinheim, 2000. doi: $10.1002 / 14356007.015$ 183 